\title{
Unusual extraction and characterization of nanocrystalline cellulose from cellulose derivatives
}

\author{
Luis Alves ${ }^{\mathrm{a}}$, Bruno Medronho ${ }^{\mathrm{b}, *}$, Filipe E. Antunes ${ }^{\mathrm{a}}$, Maria P. Fernández-García ${ }^{\mathrm{c}}$, João Ventura ${ }^{\mathrm{c}}$, João P. Araújo ${ }^{\mathrm{c}}$, \\ Anabela Romano ${ }^{\mathrm{b}}$, Bjorn Lindman ${ }^{\mathrm{d}, e, \mathrm{f}}$
}

a University of Coimbra, Department of Chemistry, 3004-535 Coimbra, Portugal

${ }^{b}$ Faculty of Sciences and Technology, Centre for Mediterranean Bioresources and Food, University of Algarve, Ed. 8, Campus de Gambelas, 8005-139 Faro, Portugal

c IFIMUP and IN - Institute of Nanoscience and Nanotechnology, Department of Physics and Astronomy, Faculty of Science, University of Porto, 4169-007 Porto, Portugal

d Division of Physical Chemistry, Department of Chemistry, Center for Chemistry and Chemical Engineering, Lund University, SE-221 00 Lund, Sweden

e Materials Science and Engineering, Nanyang Technological University, Singapore 639798, Singapore

${ }^{\mathrm{f}}$ FSCN, Fibre Science and Communication Network, MidSweden University, Sundsvall, Sweden

\section{A R T I C L E I N F O}

\section{Article history:}

Received 14 October 2014

Received in revised form 28 November 2014

Accepted 6 December 2014

Available online 12 December 2014

\section{Keywords:}

Nanocrystals

Cellulose derivatives

Hydroxypropyl methylcellulose

Carboxymethyl cellulose

\begin{abstract}
A B S T R A C T
Unlike many nanomaterials, nanocrystalline cellulose $(\mathrm{CNC})$ is not synthesized from molecular or atomic components but rather extracted from naturally occurring cellulose. Undoubtedly, the exploitation of CNCs will become a bridge between nanoscience and natural resource products, which could play a major role in reviving the forest industry. In this work, CNC was successfully extracted from unusual sources, hydroxypropyl methylcellulose (HPMC) and carboxymethylcellulose (CMC). The extracted crystallites were purified and further characterized by Fourier transform infrared (FTIR), scanning electron microscopy (SEM), X-ray powder diffraction (XRD) and dynamic light scattering (DLS). The average size of the CNCs extracted from HPMC and CMC was found to be less (and with lower zeta potential) than the ones extracted from microcrystalline cellulose (MCC). On the other hand, FTIR and XRD revealed that native HPMC and CMC are unexpectedly highly crystalline and hence can be used as a source for CNCs.
\end{abstract}

(c) 2014 Elsevier B.V. All rights reserved.

\section{Introduction}

Cellulose, which can be obtained from many sources such as wood pulp, cotton, ramie, and bacteria, is the most abundant and renewable biopolymer resource available today in nature. This polymer organizes in a rather dense and highly hierarchal fashion where an extended intra- and intermolecular network of hydrogen bonds and Van der Waals interactions are believed to constitute the basis of cohesion between cellulose molecules [1-8]. Within the cellulose fibrils there are regions where the cellulose chains are arranged in highly ordered structures - crystallites - and regions that are disordered - amorphous - [9]. The latter regions act as structural defects and can be easily accessed and removed/dissolved by treating the biopolymer with harsh acid conditions. The resulting product is the recalcitrant individual needle-like crystallites of cellulose chains which are commonly called nanocrystals (CNCs). Other related but not necessarily synonymous terminologies are often found in literature such as whiskers, microcrystalline cellulose and cellulose crystallites [10]. In the recent years, CNCs have been realized as a new class of nanomaterials attracting immense interest from both research scientists and industrialists. Compared to cellulose fibers,

\footnotetext{
* Corresponding author.

E-mail address: bfmedronho@ualg.pt (B. Medronho).
}

CNCs possess many advantages, such as nanoscale dimension, high specific strength and modulus, high surface area, and unique optical properties. These unmatched physico-chemical properties and the apparent lack of environmental constraints enable the use of CNCs in a wide range of potential applications such as a reinforcing agent in nanocomposites, coatings, textiles, aerospace and many others [9,11-13]. The morphology and properties of CNCs are strongly dependent on the source of the original cellulose, extraction procedure and their parameters $[14,15]$. Therefore, for an efficient comparison and development of these resources, the isolation and further analysis of CNCs from different cellulosic resources are particularly relevant and almost required $[13,16$, 17]. CNCs have been isolated from a variety of sources such as vegetable sources, cotton, wood pulp $[14,18]$ and agricultural byproducts, such as soy hulls [17], corncob [13] and rice husk [19], and animal sources such as tunicates and bacteria [20-24].

In this work, the cellulose derivatives hydroxypropyl methylcellulose (HPMC) and carboxymethyl cellulose (CMC) were chosen as possible sources of CNCs. The HPMC is one of the most commonly used hydrophilic biodegradable polymers for controlled release formulations since it works as a pH-independent gelling agent. On the other hand, CMC finds applications in medicine, paints, detergents and in the food domain. Both cellulose derivatives share some useful characteristics such as the high viscosity at low concentrations and defoaming, 
surfactant and bulking abilities [25]. In general, cellulose derivatives result from the non-homogeneous substitution of the hydroxyl groups in each anhydroglucose ring by other functional groups. During this process, a decrease in the crystallinity of the substance is expected to occur and therefore, at a first glance, one could argue that cellulose derivatives are not an obvious choice as a source of CNCs. However, despite the fact that hydroxypropyl and methyl groups (in HPMC) and carboxylate groups (in $\mathrm{CMC}$ ) have replaced the hydroxyls, the crystallinity of both derivatives was here found to be unexpectedly high and thus the extraction of CNCs appears as a viable process. To our knowledge, CNCs have never been extracted from cellulose derivatives and therefore we here report the initial results regarding their extraction and characterization. Scanning electron microscopy (SEM) and dynamic light scattering (DLS) were used to study the morphology while FTIR and X-ray diffraction were further used to infer about more detailed molecular information of the extracted CNCs.

\section{Experimental}

\subsection{Materials}

Microcrystalline cellulose (MCC) (Avicel PH-101, degree of polymerization ca. 260), carboxymethyl cellulose (CMC) ( Mw $=7 \times 10^{5} \mathrm{Da}$, degree of substitution of 0.9 ), and sulfuric acid (98\% purity ACS reagent), were obtained from Sigma-Aldrich. Hydroxypropyl methylcellulose (HPMC, Methocel $®$ K15M Premium), 19-24\% methoxyl and 7-12\% hydroxypropyl, $\mathrm{Mw}=4.3 \times 10^{5} \mathrm{Da}$, was purchased from Dow Chemical Company and was used without further purification. Dialysis bags (molecular weight cutoff 2000, Cellu Sep H1) were purchased from Orange Scientific. All samples were prepared using deionized water. The CNCs were extracted according to the procedure outlined below.

\subsection{Extraction of nanocrystalline cellulose}

The extraction procedure of CNCs was based on a previously reported method [26]. Briefly, $5 \mathrm{~g}$ of MCC powder was added to a $100 \mathrm{~mL}$ sulfuric acid ( $65 \mathrm{wt} . \%$ ) aqueous solution. The hydrolysis was carried out during $30 \mathrm{~min}$ at $65^{\circ} \mathrm{C}$ and afterwards the reaction was quenched by the addition of a large excess of deionized water $(250 \mathrm{~mL})$ and the mixture was centrifuged at $3800 \mathrm{rpm}$ for $15 \mathrm{~min}$ at room temperature. This centrifugation step was repeated several times before the suspension was dialyzed against distilled water for one week to neutralize the $\mathrm{pH}$ of solution and remove undesired salts. The resulting suspension was kept refrigerated for later use. The same procedure was followed for the extraction of CNCs from HPMC and CMC.

\subsection{Scanning electron microscopy}

High resolution (Schottky) Scanning Electron Microscope, equipped with the analytical systems X-ray microanalysis (EDS) and backscattered electron diffraction pattern analysis (EBSD) was used to observe the morphology and size of the extracted CNCs (model Quanta 400FEG ESEM/EDAX Genesis X4M). Generally, $50 \mu \mathrm{L}$ of a suspension (0.1 wt.\% crystals) was dropped onto clean glass lamella followed by drying for $24 \mathrm{~h}$ in a kiln and then sputtered with an approximately $6 \mathrm{~nm}$ thin Au/Pd film by cathodic pulverization using a SPI Module Sputter Coater before SEM analysis, during $90 \mathrm{~s}$ with a current of $15 \mathrm{~mA}$. Starting materials (powders) were deposited directly over the carbon tape on the support and sputtered following the procedure previously described. The accelerating voltage ranged from 5 to $15 \mathrm{kV}$.

\subsection{X-ray diffraction}

The X-ray diffraction (XRD) experiments were performed on a Siemens D5000 X-ray diffractometer, capable of identifying crystalline phases down to $3 \%$ of the bulk. This equipment consists of a $\theta / 2 \theta$ diffraction instrument. $\mathrm{A} \mathrm{CuK}_{\alpha 1}$ is used as radiation source with $\lambda=$ $1.54056 \AA$, focused by a primary Ge crystal monochromator. The detector is a standard scintillation counter. The $\mathrm{Cu}$ tube runs at $40 \mathrm{~mA}$ and $40 \mathrm{kV}$. The cooling is supplied by an internal water-filled recirculation chilling system, running at approximately $16{ }^{\circ} \mathrm{C}$ with a flow rate of $4-$ $4.5 \mathrm{~L} / \mathrm{min}$. The slit arrangement is a $2 \mathrm{~mm}$ pre-sample slit, $2 \mathrm{~mm}$ postsample slit and a $0.2 \mathrm{~mm}$ detector slit. The freeze-dried crystals (powder) were placed in a proper support and analyzed.

\subsection{Fourier transform infrared spectroscopy}

The infrared spectra were recorded at $25^{\circ} \mathrm{C}$ with an ATR-FTIR spectrophotometer Thermo Nicolet, IR300 (USA), using a universal ATR sampling accessory. FTIR spectral analysis was performed within the wave number range of $400-4000 \mathrm{~cm}^{-1}$. A total of 256 scans were run to collect each spectrum at a resolution of $1 \mathrm{~cm}^{-1}$ in the transmission mode. The CrI (also referred to as "lateral order index"-LOI) was estimated from the ratio between the absorption band at $1430 \mathrm{~cm}^{-1}$ and the absorption band at $890 \mathrm{~cm}^{-1}[27,28]$. Additionally, the "total crystallinity index" (TCI) was estimated from the ratio between the bands at $1370 \mathrm{~cm}^{-1}$ and $2900 \mathrm{~cm}^{-1}$ [29].

\subsection{Dynamic light scattering}

The potential charges on the surface of CNCs in pure water were measured with a Malvern Zetasizer Nano ZS, while the particle averaged size was determined by photon correlation spectroscopy (PCS) on the same equipment. To avoid excess light scattering in the spectrometer, the samples measured on the Zetasizer Nano ZS were diluted to a final concentration of $1.0 \mathrm{wt} . \%$ and an ultrasonic bath was used to facilitate dispersion of the CNCs in water.

\section{Results and discussion}

\subsection{Microscopy characterization: morphology and zeta potential}

The morphology of CNCs can be studied by different methods. The most common and conventional techniques to probe cellulose structure on the nanometer level are atomic force microscopy (AFM) [30] and transmission electron microscopy (TEM) [31]. The latter, among other advantages, can provide high-resolution images of the samples while the former can give topographic profiles of individual cellulose chains down to the atomic level. Besides these techniques, environmental scanning electron microscopy (ESEM) has been successfully applied [32] which, like conventional SEM, is a surface technique offering significant high spatial resolution particularly important for the study of biological samples, such as cellulose.

In Fig. 1, the SEM micrographs of the raw cellulosic materials used in this work are shown.

Coarse particles of different sizes are observed in all celluloses. Additionally, in the CMC case, soft elongated aggregates are also visible. When the dry powders are suspended in water, the crystalline cellulose sample (MCC) presents coarse particles with an average size around $50 \mu \mathrm{m}$, while the cellulose derivatives (for instance, HPMC) present particles with a smaller average size, i.e. between 2 and $5 \mu \mathrm{m}$ (Fig. 2).

The larger size of the MCC particles might also reflect some aggregation due to their lower solubility in water. On the other hand, the cellulose derivatives are water-soluble and, therefore, individual particles are expected to disperse more efficiently in the solvent.

The SEM micrographs of the CNC suspensions extracted from the cellulose derivatives CMC and HPMC are shown in Fig. 3 (panels c, d and panels e, f, respectively). For comparison, micrographs of the CNCs from the MCC sample are shown on the top of Fig. 3 (panels a and b).

The CNCs from MCC (Fig. 3, a and b) are wider (ca. 200-300 nm) and longer (ca. $1 \mu \mathrm{m}$ ) than the CNCs from the cellulose derivatives: CMC (Fig. 3, c and d) shows individual crystallites which are approximately 

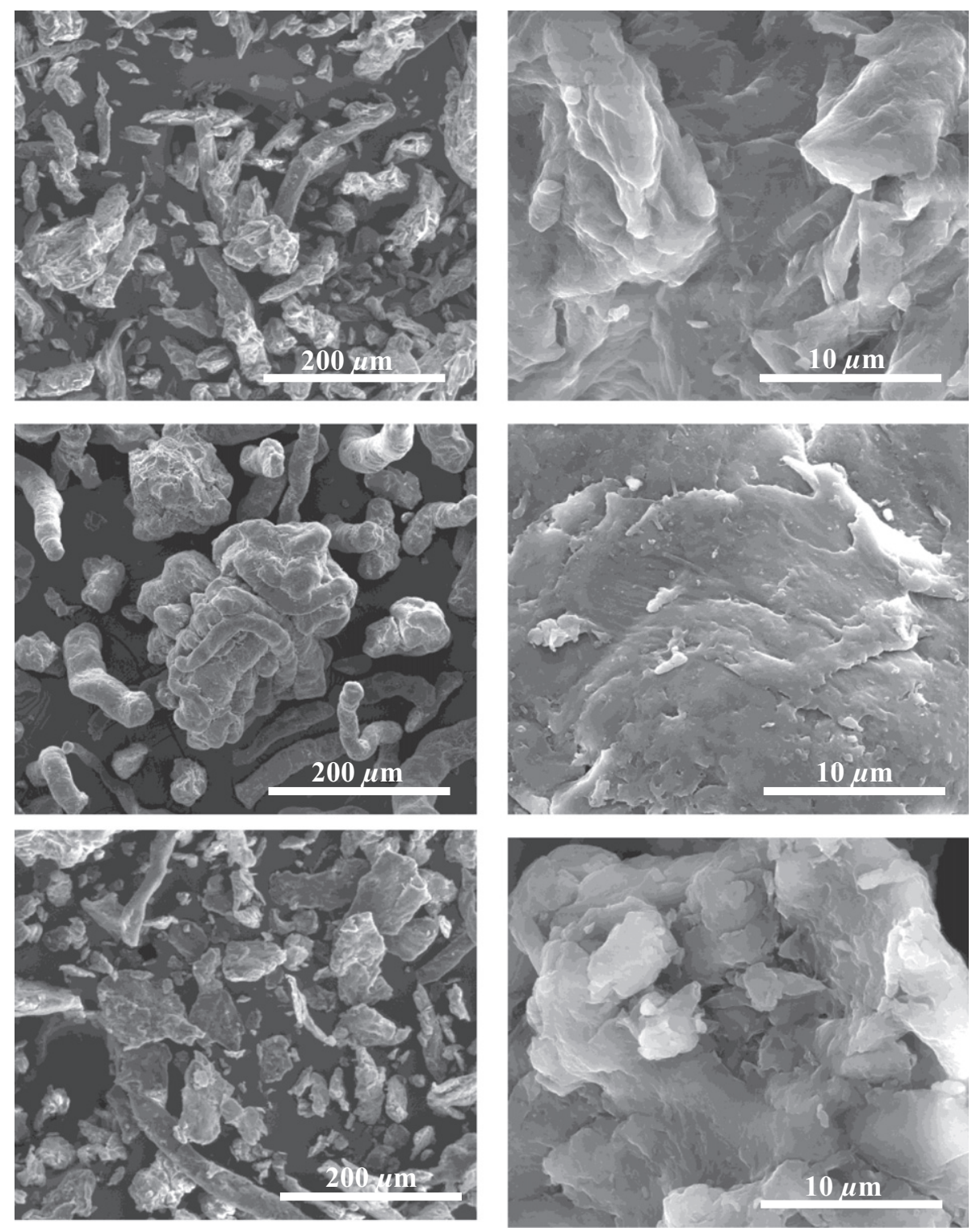

Fig. 1. SEM micrographs of native celluloses used: MCC (top), CMC (middle) and HPMC (bottom).

50-100 nm wide and 300-600 nm long while the crystallites from HPMC (Fig. 3, e and f) are approximately 50-100 nm wide and 300$400 \mathrm{~nm}$ long. In both systems, a small fraction of larger needle-like crystals can also be observed.

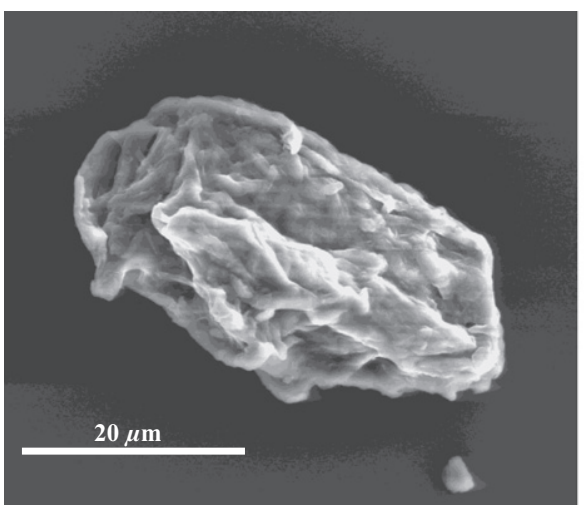

One should mention that the dimensions estimated by SEM are significantly higher than those obtained from AFM for the CNCs extracted from MCC [11]. Among other reasons, this might suggest selfassociation of rods along a single axial direction already in the solution

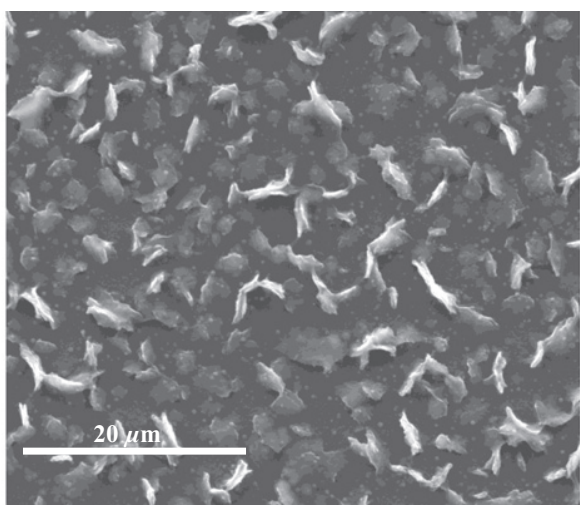

Fig. 2. SEM micrographs of MCC (left) and HPMC (right). In both cases, $0.1 \%$ cellulose was dispersed in water. 

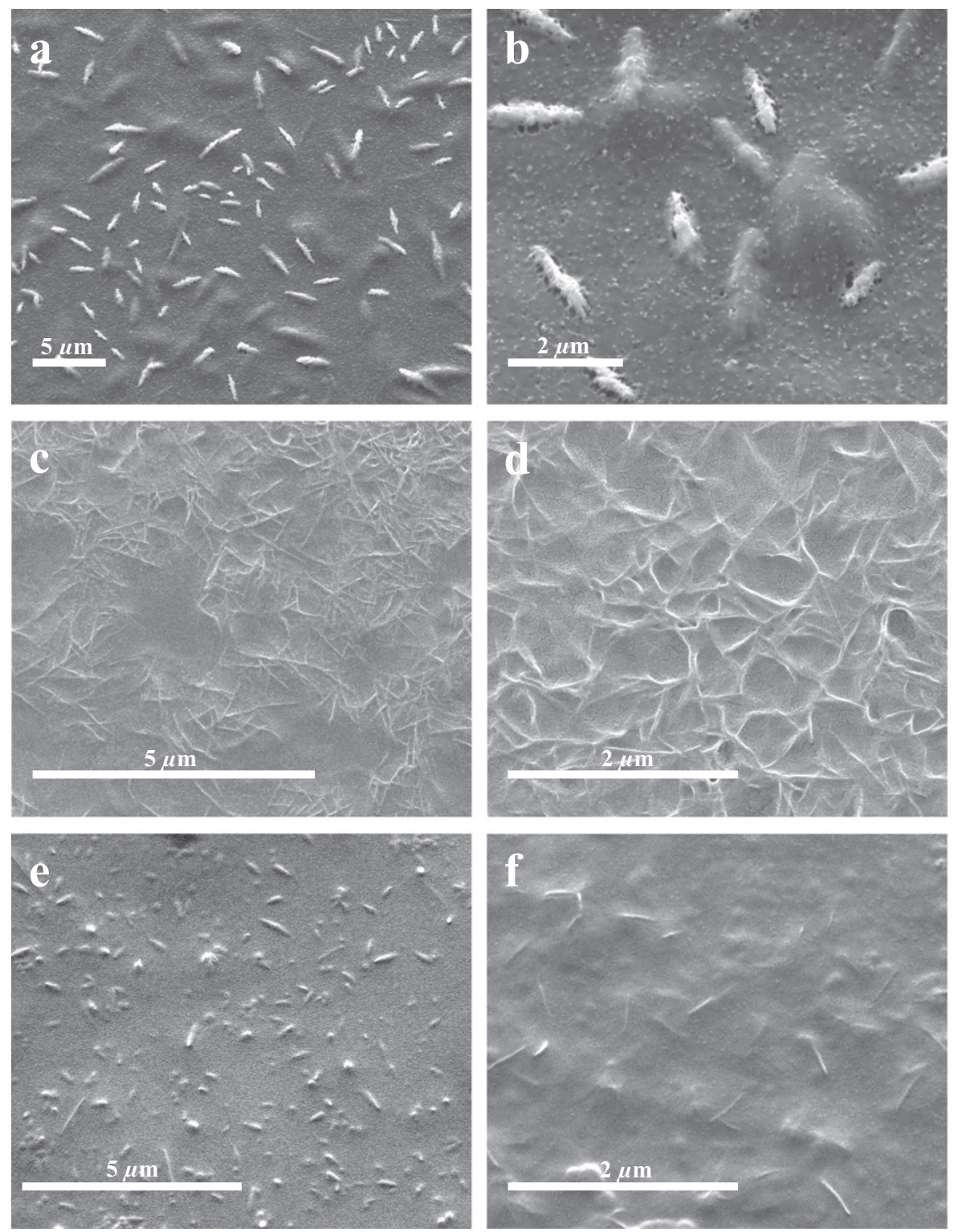

Fig. 3. SEM micrographs of CNC extracted from MCC (a and b), CMC (c and d) and HPMC (e and f).

state, or during specimen preparation for SEM. Additionally, the mechanical treatment provided to disperse CNCs as a uniform stable suspension might not be enough to separate all $\mathrm{CNC}$ rods as individual entities and thus the size might be overestimated.

CNC particle size was also estimated from photon correlation spectroscopy (PCS). Since PCS is a light-scattering method, the measured CNC particle size values are the z-average (intensity mean) hydrodynamic diameters of equivalent spheres and do not represent actual physical dimensions of the rod-like CNC particles. However, the extracted values are valid for comparison purposes. In Table 1 we can see some structural parameters derived from the different techniques used.

The first observation is that, not surprisingly, the average size estimated from PCS is smaller than the size estimated from SEM. More importantly, and in agreement with the SEM analysis, the average particle

Table 1

Characteristics (i.e. dimensions, zeta potential and CrI) of CNCs extracted from HPMC, CMC and MCC.

\begin{tabular}{|c|c|c|c|c|c|c|c|c|}
\hline & \multicolumn{2}{|c|}{$\mathrm{L}$, length (nm) } & \multirow[t]{3}{*}{ Average width $(\mathrm{d})(\mathrm{nm})^{\mathrm{a}}$} & \multirow{3}{*}{$\begin{array}{c}\text { Average aspect ratio } \\
\text { (L/d) }\end{array}$} & \multirow[t]{3}{*}{ Zeta potential $(\mathrm{mV})$} & \multicolumn{3}{|c|}{ Crystallinity } \\
\hline & \multirow[t]{2}{*}{ SEM } & \multirow[t]{2}{*}{$\overline{\text { DLS }}$} & & & & \multicolumn{2}{|c|}{ FTIR } & \multirow{2}{*}{$\begin{array}{c}\text { X-ray }{ }^{\mathrm{d}} \\
(\%)\end{array}$} \\
\hline & & & & & & $\mathrm{LOI}^{\mathrm{b}}$ & $\mathrm{TCl}^{\mathrm{C}}$ & \\
\hline $\mathrm{CNC}_{\text {HPMC }}$ & $300-400$ & 260 & $50-100$ & $3-8$ & -8.6 & $1.19(0.53)$ & $1.47(0.95)$ & $53(81)$ \\
\hline $\mathrm{CNC}_{\mathrm{MCC}}$ & 1000 & 820 & $100-300$ & $3.3-10$ & -51.5 & $0.73(0.70)$ & $0.95(0.87)$ & $77(75)$ \\
\hline $\mathrm{CNC}_{\mathrm{CMC}}$ & $300-600$ & 218 & $50-100$ & $3-6$ & -33.2 & $1.29(0.98)$ & $1.34(0.77)$ & $59(57)$ \\
\hline
\end{tabular}

\footnotetext{
a Estimated from the SEM micrographs.

b "Lateral order index" (LOI) estimated from the ratio between the vibration band at $1430 \mathrm{~cm}^{-1}$ and the band at $890 \mathrm{~cm}^{-1}$ (Ref. [21]).

c "Total crystallinity index" (TCI) estimated from the ratio of absorption bands at $1372 \mathrm{~cm}^{-1}$ and $2900 \mathrm{~cm}^{-1}$ (Ref. [23]).
}

d "Crystallinity index" estimated from the empirical Segal method (REF. [43]). 
size of the CNCs extracted from HPMC and CMC is considerably smaller than the one from MCC.

Another useful information is the aspect ratio (defined as the lengthto-diameter, $\mathrm{L} / \mathrm{d}$ ) which is an important factor normally indicated as a controlling parameter for the mechanical properties of nanocomposites. Typically, CNCs with a high aspect ratio tend to give the best reinforcing effect [11] and this parameter depends on the original cellulose characteristics and extraction conditions (i.e. temperature, acid concentration, time, etc.). Here, the $\mathrm{L} / \mathrm{d}$ values found were very similar (L/d 3-10) but in agreement with previous reports for Avicel whiskers [33].

An additional important characteristic of CNCs, when prepared in sulfuric acid, is that the particles possess negatively charged surfaces resulting from the esterification of hydroxyl groups by sulfate ions. This process is expected to enhance CNC colloidal stability in aqueous solutions due to electrostatic repulsion among charged particles. The determined zeta-potential is presented in Table 1. Essentially, the zeta-potential measures the mobility of a distribution of charged particles as they are subjected to an electric field. The CNCs extracted from the MCC present an average zeta potential of $-51.5 \mathrm{mV}$. On the other hand, the CNCs extracted from cellulose derivatives, HPMC and CMC, present an average zeta potential of $-8.6 \mathrm{mV}$ and $-33.2 \mathrm{mV}$, respectively. The lower zeta potential of the former might be important for hypothetical biocompatibility issues, however the colloidal stability of the samples is poorer and flocculation was frequently observed. This lower value of the zeta potential can be rationalized as follows; when the amorphous regions of the HPMC are hydrolyzed, the remaining crystallites are expected to preserve their structure. It is reasonable to assume that the isolated crystals possess hydroxypropyl and methyl modifications from the original HPMC and, therefore, are less susceptible for esterification when compared with the hydroxyl groups in CNCs derived from MCC (i.e. the ether modifications are more stable in acidic media than in the hydroxyls, which can be readily esterified). Thus, a higher surface charge is anticipated for the CNCs extracted from MCC while a much lower charge density is expected for the CNCs from HPMC. The same is valid for the CNCs derived from CMC with the difference that in this case even if no etherification occurs the crystallites possess already charged carboxylate groups and, therefore, the zeta potential of the CMC nanocrystals is found in-between the zeta-potentials of HPMC and MCC.

\subsection{FTIR characterization}

Fig. 4 shows the FTIR spectra of native celluloses as well as of extracted CNCs. Both native cellulose and extracted CNCs are found to be very similar with only slight differences, mainly regarding intensity.

In all cases, the characteristic bands can be identified [34,35]; the broad absorption in the range of $3100-3600 \mathrm{~cm}^{-1}$ can be ascribed to the stretching of the $-\mathrm{OH}$ groups (with typical sharpening at $3400 \mathrm{~cm}^{-1}$ ) [36-38] while the peak at $2900 \mathrm{~cm}^{-1}$ appears due to $\mathrm{C}-\mathrm{H}$ stretching $[36,38]$. An intense band between 1600 and $1650 \mathrm{~cm}^{-1}$ originates from the absorbed moisture (i.e. bending mode of water absorbed to cellulose) [39]. The deformation, wagging and twisting modes of anhydroglucopyranose vibration are shown from 600 to $1800 \mathrm{~cm}^{-1}$. More specifically, the absorbance at around $900 \mathrm{~cm}^{-1}$ can be assigned to the $\mathrm{C}-\mathrm{H}$ deformation mode of the glycosidic linkage between the glucose units $[40,41]$, while the absorbance bands between $1000 \mathrm{~cm}^{-1}$ and $1200 \mathrm{~cm}^{-1}$ are attributed mainly to the $\mathrm{C}-\mathrm{O}$ stretching in major ether bands [41]. We note that the changes observed in the zeta potential measurements are not clearly evident in the FTIR analysis mainly due to the fact that some of the expected vibrational bands from the esterification are masked by other major bands (i.e. the vibrational modes of $\mathrm{C}-\mathrm{H}$ and $\mathrm{O}-\mathrm{H}$ superimpose and dominate the spectra). Nevertheless, in some cases, we can see in the extracted crystal signs of these vibration bands such as in Fig. 4 (top spectrum of CNCs from MCC shows a small peak at around $833 \mathrm{~cm}^{-1}$ which can be assigned to the $S=0$ stretching) or the signal from the CNCs from the
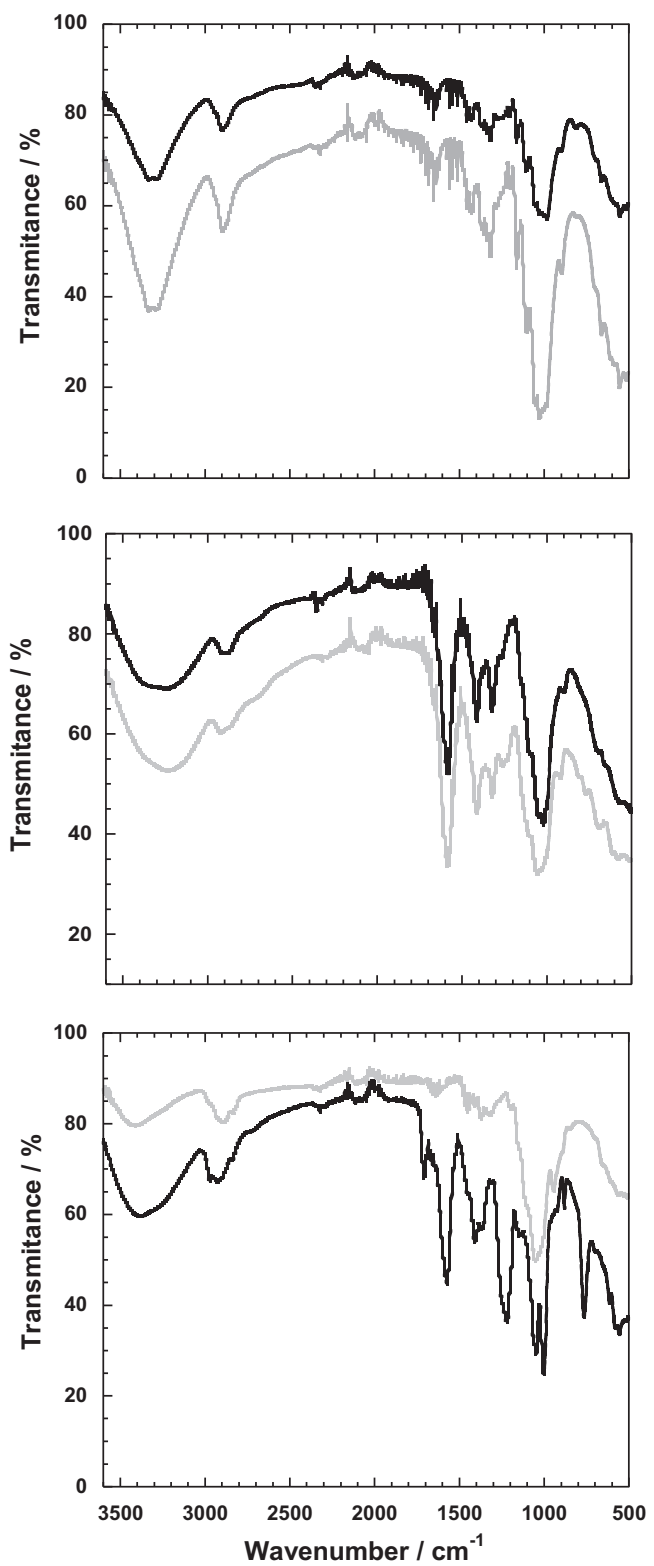

Fig. 4. FTIR spectra of a) MCC (gray line) and extracted $\mathrm{CNC}_{\mathrm{MCC}}$ (black line), b) $\mathrm{CMC}$ (gray line) and extracted $\mathrm{CNC}_{\mathrm{CMC}}$ (black line) and c) HPMC (gray line) and extracted $\mathrm{CNC}_{\mathrm{HPMC}}$ (black line).

HPMC where a band at around $1250 \mathrm{~cm}^{-1}$ can be assigned to the $\mathrm{C}-\mathrm{O}-\mathrm{S}$ stretching mode. A more complete summary of the main vibration modes is given in Table 2 .

The segments in the cellulose polymer chain will vibrate differently in well ordered crystalline phases in comparison to less ordered amorphous phases and, therefore, it is possible to assign absorption bands related to crystalline and amorphous regions and estimate a kind of crystallinity index (CrI) from FTIR. This is one of the simplest methods but one should keep in mind that the extracted values are not absolute. O'Connor et al. [27] established that the absorption band at around $1430 \mathrm{~cm}^{-1}$ is characteristic of crystalline areas in the polymer and the absorption band at $890 \mathrm{~cm}^{-1}$ typical of amorphous regions; the ratio of these two bands was established as a "crystallinity index", later referred to as the "lateral order index" (LOI). Later, Nelson and O'Connor [29] defined another crystalline parameter from the ratio of absorption bands at $1370 \mathrm{~cm}^{-1}$ and $2900 \mathrm{~cm}^{-1}$, the so called "total crystallinity index" (TCI). The TCI is said to be proportional to the crystallinity degree of cellulose while LOI is correlated to the overall degree of order in cellulose [34,35]. In Table 1, both TCI and LOI are presented. The numbers 
Table 2

Vibrational frequencies $\left(\mathrm{cm}^{-1}\right)$ of original celluloses (MCC, CMC and HPMC) and extracted $\mathrm{CNCs}\left(\mathrm{CNC}_{\mathrm{MCC}}, \mathrm{CNC}_{\mathrm{CMC}}\right.$ and $\left.\mathrm{CNC}_{\mathrm{HPMC}}\right)$.

\begin{tabular}{|c|c|c|c|c|c|c|}
\hline \multicolumn{6}{|c|}{ Frequency $\left(\mathrm{cm}^{-1}\right)$} & \multirow[t]{2}{*}{ Peak assignment } \\
\hline $\mathrm{CMC}$ & $\mathrm{CNC}_{\mathrm{CMC}}$ & HPMC & $\mathrm{CNC}_{\mathrm{HPMC}}$ & MCC & $\mathrm{CNC}_{\mathrm{MCC}}$ & \\
\hline 3280 & 3230 & 3400 & 3370 & 3300 & 3300 & $\mathrm{O}-\mathrm{H}$ str. \\
\hline 2890 & 2900 & 2900 & 2900 & 2890 & 2890 & $\mathrm{C}-\mathrm{H}$ str. \\
\hline 1500 & 1580 & 1640 & 1580 & 1650 & 1650 & $\mathrm{O}-\mathrm{H}$ def. due to $\mathrm{H}_{2} \mathrm{O}$ \\
\hline \multirow[t]{2}{*}{1410} & 1410 & 1450 & 1460 & 1430 & 1430 & $\mathrm{CH}_{2}$ def. \\
\hline & & 1370 & 1400 & 1370 & 1370 & $\mathrm{C}-\mathrm{H}$ def.; $\mathrm{COO}$ str. \\
\hline 1320 & 1320 & 1315 & 1350 & 1310 & 1310 & $\mathrm{C}-\mathrm{H}$ def.; $\mathrm{C}-\mathrm{H}$ wag; $\mathrm{O}-\mathrm{H}$ def. \\
\hline- & 1250 & 1200 & 1220 & 1210 & 1210 & $\mathrm{O}-\mathrm{H}$ str. \\
\hline- & - & - & 1150 & 1160 & 1160 & $\mathrm{C}-\mathrm{O}-\mathrm{C}$ asymmetric str \\
\hline 1020 & 1050 & 1050 & 1050 & 1100 & 1100 & anhydroglucose ring str.; $\mathrm{C}-\mathrm{O}$ str. \\
\hline- & - & - & 990 & 1000 & 990 & $\mathrm{C}-\mathrm{O}$ str.; $\mathrm{C}-\mathrm{H}$ str. \\
\hline 890 & 915 & 930 & 890 & 890 & 890 & $\mathrm{C}(1)-\mathrm{O}-\mathrm{C}(4)$ ring str.; $\mathrm{C}-\mathrm{H}$ def. \\
\hline
\end{tabular}

inside the parentheses represent the indices for the initial cellulose (MCC, CMC and HPMC). Regardless of the method of calculation, the CrI estimated from FTIR always increases for the CNCs. This is somehow expected since during the extraction and purification procedure the amorphous areas are likely removed and thus the crystallinity increases. Another important observation is that the native cellulose derivatives, CMC and HPMC, are already quite crystalline and the CNCs extracted from them present both TCI and LOI indices higher than the ones corresponding to the CNCs extracted from MCC.

This high crystallinity of the cellulose derivatives is not trivial to understand. Modifications are expected to affect adversely the good packing of cellulose chains in crystals (i.e. decrease the crystallinity and enhance solubility). This is why essentially any modification done on cellulose tends to make it more soluble in water; apart from HPMC and CMC, other notable examples are, for instance, methyl cellulose (MC) and hydroxyethylcellulose (HEC). The former is highly soluble in water, even if from a polarity point of view it would be expected to be less soluble. In the latter case, also highly soluble, substitution would not change the number of hydrogen bonds (typical argument used to explain cellulose insolubility in water) compared to unmodified cellulose. Therefore, it is somehow surprising that the (water soluble) cellulose derivatives studied here present such a high initial crystallinity. Although relevant, the data suggests that crystallinity is indeed not the critical parameter for cellulose solubility.

\subsection{X-ray characterization}

The X-ray diffraction patterns of native celluloses and extracted CNCs are represented in Fig. 5.

In line with the FTIR measurements, the first important observation is that, despite HPMC and CMC being modified polymers, it is clear that both are fully crystalline in nature as can be inferred from the diffraction peaks. While this is expected for the MCC, it is more striking for the cellulose derivatives CMC and HPMC (see discussion above). As one can see, the diffraction pattern of MCC is characterized by a major peak centered around $22.5^{\circ}(002)$ with a side peak at $20.5^{\circ}(021)$ typical for a cellulose I crystalline polymorph [42]. Other characteristic reflections for a cellulose I type structure can be found at $14.7^{\circ}(101), 16.6^{\circ}(10 \overline{1})$ and $34.7^{\circ}(040)$. The extracted CNCs have the same diffraction pattern as the native MCC. The synthesis of the cellulose derivatives changes the crystalline structure to a cellulose II type polymorph, where a diffraction pattern with a unique peak centered around $20.1^{\circ}(10 \overline{1})$ can be observed for both CMC and HPMC. On the other hand, the CNCs extracted from the cellulose derivatives also show a single peak but centered around $22.5^{\circ}$ (002) which, as previously discussed, can be attributed to a crystal structure of cellulose I type. Despite the fact that in XRD, intensity depends on several instrumental/sample conditions, such as sample mass/size and sample placement, so that intensity usually cannot be used to extract direct information through comparisons with other samples, we note that the diffraction intensities for CNCs from cellulose derivatives are considerably lower when compared with the native celluloses. This might mean that although the original material has a preferential cellulose II organization (due to the chemical synthesis), a small fraction of cellulose I is still present (but masked by the dominating cellulose II organization) and could be isolated in the CNCs. The degree of crystallinity was estimated following the method suggest by Segal et al. [43] and included in Table 1. In qualitative
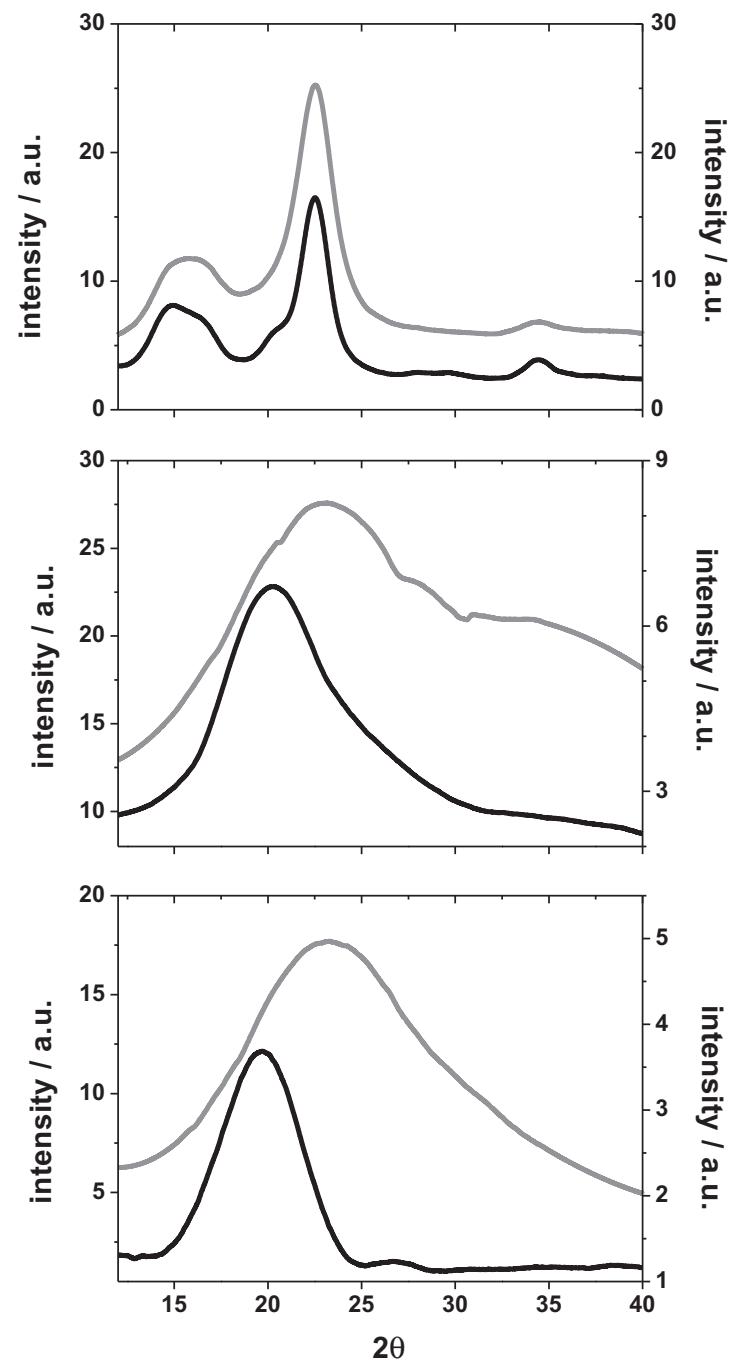

Fig. 5. XRD diffraction patterns: smoothed raw data of a) MCC (black line) and extracted $\mathrm{CNC}_{\mathrm{MCC}}$ (gray line), b) CMC (black line) and extracted $\mathrm{CNC}_{\mathrm{CMC}}$ (gray line) and c) HPMC (black line) and extracted $\mathrm{CNC}_{\mathrm{HPMC}}$ (gray line). 
agreement with the FTIR analysis, one can observe that the native cellulose derivatives are highly crystalline (the estimated $\mathrm{CrI}$ is in all cases above $50 \%$ ) and the extracted CNCs, with exception of the HPMC case, show a higher $\mathrm{CrI}$ value than the original cellulose derivatives.

\section{Conclusions}

A successful extraction and characterization of CNCs from the cellulose derivatives CMC and HPMC is reported. Surprisingly, the cellulose derivatives were found to be highly crystalline indicating an unusual extraction of CNCs. The average size of the CNCs extracted from HPMC and CMC was found to be smaller (and with lower zeta potential) than the ones extracted from microcrystalline cellulose (MCC). The estimated crystallinity indices show that the extracted material is more crystalline that the native one. However, while the MCC and CNCs share the same crystalline organization (cellulose I polymorph), the native cellulose derivatives were found to be of cellulose II type. The extracted CNCs from the cellulose derivatives show a crystalline organization of cellulose I type, which probably indicates that the starting material has a small fraction of cellulose I (masked in the diffraction spectra by the main cellulose II diffraction pattern), which is the one isolated after dissolution in the acidic media and purification.

\section{Acknowledgements}

The authors acknowledge support from the Portuguese Foundation for Science and Technology (FCT, projects PTDC/AGR-TEC/4049/2012 and FEDER/POCTI/n2-155/94, PhD and post-doc grants assigned to Luis Alves: SFRH/BD/80556/2011, Bruno Medronho: SFRH/BPD/74540/ 2010 and Maria Paz Fernández: FRH/BPD/87430/2012, respectively). Dr. Rui Rocha (CEMUP) is acknowledged for carrying out the scanning electron microscopy work.

\section{References}

[1] B. Medronho, A. Romano, M.G. Miguel, L. Stigsson, B. Lindman, Cellulose 19 (2012) 581.

[2] B. Medronho, B. Lindman, Curr. Opin. Colloid Interface Sci. 19 (2014) 32.

[3] I. Diddens, B. Murphy, M. Krisch, M. Müller, Macromolecules 41 (2008) 9755.

[4] O. Biermann, E. Hadicke, S. Koltzenburg, F. Muller-Plathe, Angew. Chem. Int. Ed. 40 (2001) 3822.
[5] C. Yamane, T. Aoyagi, M. Ago, K. Sato, K. Okajima, T. Takahashi, Polym. J. 38 (2006) 819.

[6] H. Miyamoto, M. Umemura, T. Aoyagi, C. Yamane, K. Ueda, K. Takahashi, Carbohydr. Res. 344 (2009) 1085

[7] M. Bergenstrahle, K. Mazeau, L.A. Berglund, Eur. Polym. J. 44 (2008) 3662.

[8] H.M. Cho, A.S. Gross, J.W. Chu, J. Am. Chem. Soc. 133 (2011) 14033.

[9] R.J. Moon, A. Martini, J. Nairn, J. Simonsen, J. Youngblood, Chem. Soc. Rev. 40 (2011) 3941.

[10] G. Siqueira, S. Tapin-Lingua, J. Bras, D.D. Perez, A. Dufresne, Cellulose 18 (2011) 57.

[11] B.L. Peng, N. Dhar, H.L. Liu, K.C. Tam, Can. J. Chem. Eng. 89 (2011) 1191.

[12] P. Podsiadlo, S.Y. Choi, B. Shim, J. Lee, M. Cuddihy, N.A. Kotov, Biomacromolecules 6 (2005) 2914.

[13] H.A. Silverio, W.P.F. Neto, N.O. Dantas, D. Pasquini, Ind. Crop. Prod. 44 (2013) 427.

[14] S. Beck-Candanedo, M. Roman, D.G. Gray, Biomacromolecules 6 (2005) 1048.

[15] S. Elazzouzi-Hafraoui, Y. Nishiyama, J.L. Putaux, L. Heux, F. Dubreuil, C. Rochas, Biomacromolecules 9 (2008) 57.

[16] W.S. Chen, H.P. Yu, Y.X. Liu, Y.F. Hai, M.X. Zhang, P. Chen, Cellulose 18 (2011) 433.

[17] W.P.F. Neto, H.A. Silverio, N.O. Dantas, D. Pasquini, Ind. Crop. Prod. 42 (2013) 480.

[18] E.D. Teixeira, A.C. Correa, A. Manzoli, F.L. Leite, C.R. de Oliveira, L.H.C. Mattoso, Cellulose 17 (2010) 595.

[19] S.M.L. Rosa, N. Rehman, M.I.G. de Miranda, S.M.B. Nachtigall, C.I.D. Bica, Carbohydr Polym. 87 (2012) 1131.

[20] O. van den Berg, J.R. Capadona, C. Weder, Biomacromolecules 8 (2007) 1353.

[21] J. Araki, S. Kuga, Langmuir 17 (2001) 4493.

[22] M. Roman, W.T. Winter, Biomacromolecules 5 (2004) 1671.

[23] V. Favier, H. Chanzy, J.Y. Cavaille, Macromolecules 28 (1995) 6365.

[24] L. Heux, G. Chauve, C. Bonini, Langmuir 16 (2000) 8210.

[25] D. Klemm, B. Heublein, H.P. Fink, A. Bohn, Angew. Chem. Int. Ed. 44 (2005) 3358.

[26] M.A. Martins, E.M. Teixeira, A.C. Correa, M. Ferreira, L.H.C. Mattoso, J. Mater. Sci. 46 (2011) 7858

[27] R.T. O'Connor, E.F. DuPré, D. Mitcham, Text. Res. J. 28 (1958) 382.

[28] F.G. Hurtubise, H. Krasig, Anal. Chem. 32 (1960) 177.

[29] M.L. Nelson, R.T. O'Connor, J. Appl. Polym. Sci. 8 (1964) 1325.

[30] L. Pranger, R. Tannenbaum, Macromolecules 41 (2008) 8682.

[31] J. Araki, M. Wada, S. Kuga, T. Okano, Colloids Surf. A 142 (1998) 75

[32] A.F. Miller, A.M. Donald, Biomacromolecules 4 (2003) 510.

[33] M.A.S.A. Samir, F. Alloin, A. Dufresne, Biomacromolecules 6 (2005) 612.

[34] J. Siroky, R.S. Blackburn, T. Bechtold, J. Taylor, P. White, Cellulose 17 (2010) 103.

[35] M. Poletto, V. Pistor, R.M.C. Santana, A.J. Zattera, Mater. Res.-Ibero Am. J. 15 (2012) 421.

[36] M.S. Jahan, A. Saeed, Z.B. He, Y.H. Ni, Cellulose 18 (2011) 451.

[37] F. Peng, J.L. Ren, F. Xu, J. Bian, P. Peng, R.C. Sun, J. Agric. Food Chem. 57 (2009) 6305

[38] R. Sun, X.F. Sun, G.Q. Liu, P. Fowler, J. Tomkinson, Polym. Int. 51 (2002) 117

[39] J.L. Ren, R.C. Sun, C.F. Liu, Z.Y. Chao, W. Luo, Polym. Degrad. Stab. 91 (2006) 2579.

[40] M. Kacurakova, A. Ebringerova, J. Hirsch, Z. Hromadkova, J. Sci. Food Agric. 66 (1994) 423.

[41] Z.H. Liu, Y.H. Ni, P. Fatehi, A. Saeed, Biomass Bioenergy 35 (2011) 1789.

[42] C.Y. Liang, Instrumental Analysis of Cotton Cellulose and Modified Cotton Cellulose, in: R.T. O'Connor (Ed.), Marcell Dekker Inc., New York, 1972, p. 59.

[43] L. Segal, J.J. Creely, A.E. Martin, C.M. Conrad, Text. Res. J. 29 (1959) 786. 